\title{
Implicit and Explicit Stances in Logic
}

\author{
Johan van Benthem ${ }^{1,2,3}$ (D)
}

Received: 17 December 2017 / Accepted: 24 September 2018 / Published online: 16 November 2018

(C) The Author(s) 2018

\begin{abstract}
We identify a pervasive contrast between implicit and explicit stances in logical analysis and system design. Implicit systems change received meanings of logical constants and sometimes also the notion of consequence, while explicit systems conservatively extend classical systems with new vocabulary. We illustrate the contrast for intuitionistic and epistemic logic, then take it further to information dynamics, default reasoning, and other areas, to show its wide scope. This gives a working understanding of the contrast, though we stop short of a formal definition, and acknowledge limitations and borderline cases. Throughout we show how awareness of the two stances suggests new logical systems and new issues about translations between implicit and explicit systems, linking up with foundational concerns about identity of logical systems. But we also show how a practical facility with these complementary working styles has philosophical consequences, as it throws doubt on strong philosophical claims made by just taking one design stance and ignoring alternative ones. We will illustrate the latter benefit for the case of logical pluralism and hyper-intensional semantics.
\end{abstract}

Keywords Logic $\cdot$ Modality · Implicit · Explicit · Vocabulary · Translation

\section{Explicit and Implicit Stances in Logical Analysis}

The history of logic has themes running from description of ontological structures in the world to elucidating patterns in inferential or communicative human activities.

Johan van Benthem

johan@stanford.edu

http://staff.fnwi.uva.nl/j.vanbenthem

1 Institute for Logic, Language and Computation, University of Amsterdam, P.O. Box 94242,

1090 GE Amsterdam, The Netherlands

2 Department of Philosophy, Stanford University, Stanford, CA, USA

3 Department of Philosophy, Tsinghua University, Beijing, China 
For both strands, the mathematical foundational era added a methodology of formal systems with semantic notions of truth and validity and matching proof calculi. This modus operandi is standard fare, enshrined in the major systems of the field.

Live disciplines are not finished fields but advancing quests. Logic has a growing agenda, including the study of information, knowledge, belief, action, agency, and other key topics in philosophical logic or computational logic. How are such topics to be brought into the scope of the established mathematical methodology? There are both modifications and extensions of classical logic for these purposes, and the aim of this paper is to point at two main lines, representing a significant contrast.

A major line of enriching classical logic adds new operators for new notions to be analyzed, leaving old explanations of existing logical notions untouched. A typical case is modal logic, adding operators for modalities, while nothing changes in the propositional base logic. Let us call this the explicit style of analysis, though the label 'conservative' makes sense, too: we do not touch notions already in place.

But there is also another line, where we use new concepts to modify or enrich our understanding of what the old logical constants meant, or what the old notion of valid consequence was meant to do. This leads to non-standard semantics, perhaps rethinking truth as 'support' or 'forcing', and to alternative logics whose laws differ from those of classical logic on the original vocabulary of connectives and quantifiers. Here the richer setting is reflected primarily, not in new laws for new vocabulary, but in deviations on reasoning patterns stated in the old language - where failures of old laws may well be significant and informative. A paradigmatic case for this approach is intuitionistic logic, but further instances keep emerging all the time. Let us call this the implicit style of analysis, without any pejorative connotation. Implicitness is a hall-mark of civilized intercourse.

We will discuss a sequence of illustrations displaying the contrast, and analyze what makes it tick. We set the scene by recalling some key facts about two wellknown systems: epistemic logic and intuitionistic logic, presented with a focus on information and knowledge. After that we discuss less standard cases such as logics of information update, default reasoning, games, quantum mechanics, and truth making. Throughout, we take explicit and implicit approaches seriously as equally natural stances, and we discuss new logical questions suggested by their co-existence. Our final conclusion from all this will be that the interplay of the two stances needs to be grasped and appreciated, as it raises new points and open problems concerning the architecture of logic, while it also has philosophical repercussions. ${ }^{1}$

Caveat We claim that the contrast highlighted in this paper makes sense, but we do not claim that it is clear-cut in all cases. For instance, implicit systems, too, can introduce new vocabulary, for logic-internal reasons that we will discuss in more detail later, and there are also systems like intuitionistic modal logic with both explicit and implicit features. There are also presuppositions to our style of analysis. The contrast as developed in this paper needs a common ground for comparing logics, and we locate that in a model-theoretic, rather than a proof-theoretic setting. Moreover, the above description of the contrast is not rhetorically neutral, as it takes classical

\footnotetext{
${ }^{1}$ The intended contrast is primarily one in design procedures, but often, it also applies to the systems produced. Still, there are delicate issues in classifying systems per se, to be discussed later on.
} 
logic as a reference point, making intuitionistic logic the rebel changing the rules. But in another world, classical logic might have been the late-comer challenging intuitionist orthodoxy. A more neutral description of the explicit/implicit contrast will be discussed at the end of the paper, after we have gone through our examples. We will also discuss several presuppositions and challenges in Section 15. Of course, a broad methodological distinction can be fruitful and illuminating even if it leads to contentious issues and borderline cases.

This may not be an easy paper to classify qua style or results, but we hope that the reader will benefit from looking at logical system design in our broad manner.

\section{Information, Knowledge, and Epistemic Logic}

A natural addition to the heartland of logic are notions of knowledge and information for agents, that have been part of the discipline from ancient times until today, $[9,37]$. In what follows we do not need intricate contemporary logics for epistemology, [35], interesting and innovative though these are. The contrast in modus operandi we are after can be seen at much simpler level, dating back to the 1960s.

Here is a major explicit way of taking knowledge and information seriously. We add modal operators for knowledge to propositional logic, and study the laws of the resulting epistemic logics on top of classical logic. These conservative operator extensions of classical logical systems have interesting structure and modeling power, also for notions beyond knowledge, such as belief.

In more detail, the classic [34] proposes an analysis of knowledge that involves an intuitive conception of information as a range of candidates for the real situation ('world', 'state'). This range may be large, and we know little, or small (perhaps as a result of prior information updates eliminating possibilities) and then we know a lot. In this setting, an agent knows that $\varphi$ at a current world $s$ if $\varphi$ is true in all worlds in the current range of $s$, the epistemically accessible worlds from $s$ via a binary relation $s \sim t$. To express reasoning in a matching syntax, we take standard propositional logic as a base, and add a clause for formulas of the form $K \varphi-$ subscripted to $K_{i} \varphi$ for different indices $i$ in case we want to distinguish between different agents $i$. Then the preceding intuition becomes the following truth definition:

$$
\begin{array}{lll}
\boldsymbol{M}, \mid=p & \text { iff } & s \in V(p) \\
\boldsymbol{M}, s \mid=\neg \varphi & \text { iff } & \text { not } \boldsymbol{M}, s \mid=\varphi \\
\boldsymbol{M}, s \mid=\varphi \wedge \psi & \text { iff } & \boldsymbol{M}, s \mid=\varphi \text { and } \boldsymbol{M}, s \mid=\psi \\
\boldsymbol{M}, s=K \varphi & \text { iff } & \boldsymbol{M}, t \mid=\varphi \text { for all } t \text { with } s \sim t .
\end{array}
$$

This extends classical propositional logic: the base clauses are standard, with one operator clause added. Epistemic accessibility is often taken to be an equivalence relation - but we can vary this if needed. The resulting logic is $S 5$ for each single agent, without non-trivial bridge axioms relating knowledge of different agents. Thus, basic epistemic logic is a conservative extension of classical logic, and the same holds for its variations like $S 4$ or $S 4.2$ that encode further intuitions concerning knowledge, [54]. In fact, it is epistemic $S 4$ that will be our base for comparison with 
intuitionistic logic later on. More intricate laws hold for modalities of common or distributed knowledge in groups, but again these will not be needed here.

Few people today see the epistemic modality as a conclusive analysis for the full philosophical notion of knowledge. But even so, this system is a perfect fit for another basic notion, the 'semantic information' that an agent has at her disposal, cf. the classic source [8]. And, the simple perspicuous explicit syntax of epistemic logic is still in wide use as a lingua franca for framing epistemological debates, for instance, for or against such basic principles of reasoning about knowledge as

$$
\begin{array}{ll}
\text { omniscience, or closure } & K(\varphi \rightarrow \psi) \rightarrow(K \varphi \rightarrow K \psi) \\
\text { introspection } & K \varphi \rightarrow K K \varphi
\end{array}
$$

Significantly, these are debates about intuitively acceptable reasoning principles for knowledge, not about the laws of the underlying propositional logic.

More sophisticated philosophical accounts define knowledge as a notion involving structure beyond mere semantic ranges, such as relevance order, plausibility order of worlds for belief (which we discuss later on), or similarity order for conditionals. Even so, logics for these extended settings tend to be multi-modal systems, that is, they still fall under what we have called the explicit approach. All this is typical for many areas of philosophical logic, such as temporal, deontic, or conditional logic. ${ }^{2}$

\section{Intuitionistic Logic}

Next, consider a second way of taking knowledge and information seriously, which is sometimes presented as a revolt against classical logic. We no longer take the old notions for granted, but redefine the meanings of the logical constants, perhaps also the notion of consequence, to get at crucial aspects of knowledge.

A typical instance of this second approach is intuitionistic logic that does not add knowledge syntax, but encodes behavior of knowledge in its deviations from the laws of classical consequence. ${ }^{3}$ This approach seems more radical, as breaking the classical laws has an iconoclastic appeal, and more subtly, the absence of explicit expressions for epistemic notions makes the behavior of knowledge now show, not in new laws, but implicitly, in absence of old laws, or in modifications of such laws. For instance, the well-known intuitionistic failure of Excluded Middle $\varphi \vee \neg \varphi$ tells us something essential about the incompleteness, in general, of our knowledge. But on the positive side, the continued intuitionistic validity of $\neg \varphi \leftrightarrow \neg \neg \neg \varphi$ reveals a more delicate form of introspection for knowledge than the simple $S 4$ law we had above - where negation now talks about knowledge in an implicit manner.

\footnotetext{
${ }^{2}$ This brief exposition may be misleading about the agenda of the field. Epistemic logic has come into wider use in computer science, game theory and linguistics because of its potential for describing multiagent interactions in communication or games. See the Handbook [21] for the state of the art.

${ }^{3}$ This is only one view of intuitionistic logic, compatible with the more epistemic strands in its genesis. On a prominent alternative view, intuitionistic logic is about a non-classical notion of truth. We will briefly discuss even a third perspective on intuitionistic logic in Section 4.
} 
Intuitionistic logic arose in the analysis of constructive mathematical proof, with logical constants acquiring their meanings in proof rules via the Brouwer-HeytingKolmogorov interpretation. In the 1950s, Beth and Kripke proposed models over trees of finite or infinite sequences, and in line with the idea of proof as establishing a conclusion, intuitionistic formulas are true at a node of such a tree when 'verified' in some intuitive sense. A general topological framework for placing all these ideas uniformly is presented in [17]. A standard version that suffices for our purposes here uses partially ordered models $\boldsymbol{M}=(W, \leq, V)$ with a valuation $V$, setting:

$$
\begin{array}{lll}
\boldsymbol{M}, s \mid=p & \text { iff } & s \in V(p) \\
\boldsymbol{M}, s \mid=\varphi \wedge \psi & \text { iff } & \boldsymbol{M}, s \mid=\varphi \text { and } \boldsymbol{M}, s \mid=\psi \\
\boldsymbol{M}, s \mid=\varphi \vee \psi & \text { iff } & \boldsymbol{M}, s \mid=\varphi \text { or } \boldsymbol{M}, s \mid=\psi \\
\boldsymbol{M}, s \mid=\neg \varphi & \text { iff } & \text { for no } t \geq s, \boldsymbol{M}, t \mid=\varphi \\
\boldsymbol{M}, s \mid=\varphi \rightarrow \psi & \text { iff } & \text { for all } t \geq s, \text { if } \boldsymbol{M}, t \mid=\varphi, \text { then } \boldsymbol{M}, t \mid=\psi
\end{array}
$$

In such partial orders, we can think of the objects $s$ as information stages or information pieces, while models unraveled to trees give a temporal picture of a record of possible investigations. Next, in line with the idea of accumulating certainty in the process of inquiry, the valuation $V$ in these models is persistent, i.e.,

$$
\text { if } \boldsymbol{M}, s \mid=p \text { and } s \leq t \text {, then also } \boldsymbol{M}, t \mid=p
$$

The truth definition as stated here lifts this persistence property to all formulas $\varphi$.

In this modus operandi, in contrast with epistemic logic, there is no separate syntax for knowledge or information - but old logical constants are re-interpreted, making negation and implication sensitive to the information structure of new models with an inclusion order that is absent in models for classical logic. In particular, an intuitionistic negation $\neg \varphi$ says that the formula $\varphi$ is not just 'not true', but refuted: in that it will never become true at any further stage along the inclusion ordering. Also, failure of classical definability equivalences leads to fine-structure for classical notions like implication, which can now be viewed in several non-equivalent ways.

This 'meaning loading' of the classical operators makes the intuitionistic laws for negation and implication deviate from classical logic. Now earlier points become precise. Famously, this semantics invalidates the law of Excluded Middle $\varphi \vee \neg \varphi$, as this disjunction fails at states where $\varphi$ is not yet verified though it will later become so. These deviations from classical logic are informative in telling us implicitly about properties of knowledge. Failure of Excluded Middle says that agents cannot decide everything a priori. Thus meaning loading makes the remaining validities more informative (they now say something new), and more mysteriously, it packs further information in the absence of classical laws - like dogs that do not bark in the night-time.

At the same time, even though the classical language is not extended in intuitionistic logic, there is an increase in expressive power, precisely because classical laws fail. For instance, $\varphi \rightarrow \psi$ is not equivalent to its classical equivalents $\neg \varphi \vee \psi$ or $\neg(\varphi \wedge \neg \psi)$ : intuitionistic logic has at least three candidates for plausible notions of 
implication. This 'splitting' may be seen as an implicit counterpart to the language extensions found in explicit approaches - an issue to which we will return below. ${ }^{4}$

\section{The Explicit/Implicit Contrast: Epistemic Versus Intuitionistic Logic}

So, now we have encountered two major research paradigms in the field of logic, both capable of taking information and knowledge seriously - but doing so in very different ways. Let us highlight the major differences that showed in the above:

epistemic logic explicit, conservative language extension of classical logic

intuitionistic logic implicit, meaning change old language, non-classical logic

Highlighting the distinction, consider the fact that we do not know the answer to every question, and maybe never will. This showed as follows in intuitionistic logic. Excluded Middle $\varphi \vee_{\text {int }} \neg_{\text {int }} \varphi$ was not valid - where indices highlight the fact that the failure occurs on the intuitionistic understanding of negation and disjunction though special cases of this principle may, and do, remain valid. In contrast with this, the law of Excluded Middle is unrestrictedly valid in epistemic logic, but it should not be confused with the invalid epistemic formula $K \varphi \vee$ class $K \neg$ class $\varphi$.

We now proceed to highlight a few presuppositions of our style of analysis.

The Platform of Comparison When saying that intuitionistic logic is non-classical, we refer to a set of laws that can be looked at purely syntactically: intuitionistic logic has fewer axioms and proof rules than classical logic. But the points about meaning referred to a semantics. This is significant. In order to compare the two systems in greater depth, we needed a common ground, viz. a class of models where both can be interpreted. The above models were one such choice, and they were presented as a shared perspective on knowledge and information, in terms of stages of inquiry ordered by temporal progression. Epistemic and intuitionistic logic then differ in how they reflect this shared structure. All our further examples in this paper will have this presupposition, we compare on a shared semantic base.

This choice of platform and stage-setting is not unique. One can generalize the above semantics to topological models of information pieces ordered by inclusion, and find the same contrast. But one can also start with models for epistemic logic that make less sense intuitionistically, say, the $S 5$-style information ranges in Sections 2 and 7 that only represent what agents know right now. The perspective of future inquiry, crucial to intuitionistic semantics, will then come in by studying temporal progression of such static information models, in ways that we will discuss later. ${ }^{5}$

\footnotetext{
${ }^{4}$ This brief exposition of intuitionistic logic does not do justice to its deep connections with proof theory, universal algebra, and category theory, or to the surprising effects of working in mathematical theories on top of a weaker base logic. See the encyclopedic source [55] for a richer story.

${ }^{5} \mathrm{~A}$ comparison with intuitionistic logic will involve adding informational actions, or a temporal logic, decomposing the earlier $S 4$-modality of global knowing through time into "always knowing locally". A technical implementation would be an embedding of $S 4$ into a bimodal temporalized $S 5$, [13].
} 
An Alternative View: Generalized Semantics A further presupposition of our comparison is that epistemic and intuitionistic logic were viewed as sharing a concern with knowledge as an external theme to be addressed. But one can also construe things in a logic-internal way. What if we view intuitionistic logic as a weakening of classical logic, emerging in a search for a safer core inside classical reasoning? Then the above models also exemplify a standard methodology: weaker alternative logics are often analyzed model-theoretically by generalizing some existing semantics. Intuitionistic stage models have classical models as the special case of endpoints where inquiry is over, but they offer many more counter-examples to validities.

Typically, generalized semantics add new structure of various kinds to old models. This suggests introducing new vocabulary for defining that structure and reasoning with it. But then a non-classical logic, too, can in principle generate new vocabulary beyond that of classical logic, challenging our description so far of the explicit/ implicit contrast. However, our earlier analysis still makes sense. If we take the newly found semantic models seriously, they become objects of independent interest for design of logics, and our two options return. Both implicit and explicit approaches can access the new structure, but they differ on whether the classical logic base stays unchanged, and thus on the nature and purpose of the new vocabulary.

Much more can be said about intuitionist and epistemic approaches to knowledge and information. But for the purposes of this paper, we just stipulate that both are based on stable sets of intuitions, both have generated a rich mathematical theory, and both seem bona fide instances of a logical modus operandi in system design.

With this first illustration, we hope to have drawn the contours of the methodological contrast we are after. In the following sections, we explore the 'implicit' versus 'explicit' divide in other cases, adding more depth to what it involves.

\section{Choice or Co-Existence: Translations and Merges}

But first it may seem time for a choice. Is intuitionistic logic or epistemic logic better or deeper as an analysis of information and knowledge? Should we prefer one over the other? Many philosophers think in this style, but we feel that this adversarial attitude is not very productive, and it also runs counter to known mathematical facts about system connections (for a similar, but more general criticism, cf. [32]).

Already in Gödel's seminal [29], there is a faithful translation from intuitionistic logic into the modal logic $S 4$ whose underlying intuition follows the present knowledge perspective. We now look at this connection to see what it achieves.

Translating IL Into EL The Gödel translation $\boldsymbol{t}$ turns the intuitionistic truth definition into a syntactic recipe, according to the following recursive clauses:

$$
\boldsymbol{t}(p)=\square p
$$

where the modal formulas $\square p$ are upward persistent on pre-orders,

$$
\begin{aligned}
& \boldsymbol{t}(\varphi \wedge \psi)=\boldsymbol{t}(\varphi) \wedge \boldsymbol{t}(\psi) \\
& \boldsymbol{t}(\varphi \vee \psi)=\boldsymbol{t}(\varphi) \vee \boldsymbol{t}(\psi)
\end{aligned}
$$




$$
\begin{aligned}
\boldsymbol{t}(\neg \varphi) & =\square \neg \boldsymbol{t}(\varphi) \\
\boldsymbol{t}(\varphi \rightarrow \psi) & =\square(\boldsymbol{t}(\varphi) \rightarrow \boldsymbol{t}(\psi))
\end{aligned}
$$

For the standard proof system $I L$ of intuitionistic propositional logic, we then have:

Fact $\quad I L \mid-\varphi \quad$ iff $\quad S 4 \mid \boldsymbol{t}(\varphi)$, for all propositional formulas $\varphi$.

This explains key features of intuitionistic logic in modal terms. For instance, varieties of implication place different demands on knowledge: intuitionistic $\varphi \rightarrow \psi$ is $\square(\varphi \rightarrow \psi)$, the earlier $\neg \varphi \vee \psi$ is the stronger $\square \neg \varphi \vee \psi$, and $\neg(\varphi \wedge \neg \psi)$ the weaker $\square(\varphi \rightarrow \diamond \psi)$. Also, intuitionistic laws like $\neg \varphi \leftrightarrow \neg \neg \neg \varphi$ are special cases of the fact that $S 4$ has 14 non-equivalent iterations of modalities. But intuitively, the modal setting is richer, as it also supports reasoning about non-persistent formulas that can become false at later stages. Thus, its view of inquiry allows for revision, not just cumulative update.

Uses of Translations Some people view translations like this as mere tricks, especially, those who see different logics as separate religions. But the translation facilitates a resounding transfer: everything an intuitionist says or infers can be understood by a classical modal logician. This facilitates traffic of ideas between intuitionistic and epistemic logic, and meaningful contacts between their agendas. For instance, key properties of $S 4$ such as decidability carry over automatically to intuitionistic logic, and applications keep emerging, such as uses of modal bisimulation in intuitionistic logic, [44]. But also conceptually, ideas from epistemic logic can now enter intuitionistic logic, such as the study of multi-agent scenarios.

Translating EL Into IL The discussion so far may have given the edge to epistemic logic, as it embeds intuitionistic logic. What about the other way around? Intuitively, as we noted, the semantics of $S 4$ seems richer, allowing non-persistent notions, but the two logics have the same computational complexity (their SAT problems are Pspace-complete), so there is no a priori obstacle to mutual translation. In fact, surprisingly, [24] gave a converse translation (with a correction in [30]), which is much less known. It works quite differently from Gödel's $\boldsymbol{t}$, by mimicking evaluation of modal formulas in finite models inside the intuitionistic language.

Thus, translations between stances occur, and they are significant as manuals for communication and interaction. So, are implicit intuitionistic and explicit epistemic logic then just the same system in different guises because of their faithful mutual embeddings? This question raises delicate issues of system identity.

Translation and System Identity Despite the clear benefits of translations, they need not reduce one logic to another in every relevant aspect. The Gödel translation encodes one particular modal take on the logical constants, which may not be what an intuitionist considers their essence. And there is more. To let the Gödel translation be faithful, deductive power must be restricted to $S 4$ or logics close to it. This is relevant: for instance, the Gödel embedding does not work for an epistemic logic like S5: $I L$ is Pspace-complete, and hence more complex than S5, which is merely $N P$-complete. And also conversely, studying the syntactical details of the encoding 
from $E L$ into $I L$, one does not get a feeling of strong resemblance between the two systems: it seems more like a case of intuitionistic logic hatching $S 4$ eggs. ${ }^{6}$

Caveat Understanding logical system identity is important, but in this paper, we want to stay away from this broader and unresolved issue. In particular, mutual translation, though a strong bond, need not imply system equivalence in all relevant aspects. One might impose stronger technical restrictions from the literature, such as idempotence of pairs of translations, or yet other category-theoretic criteria. However, in what follows, we stick with concrete examples, always keeping an open eye for intensional differences between even tightly mutually translated systems. ${ }^{7}$

From Translating to Merging Finally, moving away from an emphasis on reduction, there is a weaker but still significant contact between explicit and implicit logics, namely that of compatibility. Can such systems be merged in meaningful ways? Intuitionistic modal logics have long existed (for a recent epistemic modal logic, see [3]), and hybrids of explicit and implicit logics keep emerging, as we will see later on. Often this juxtaposition seems routine, but hybrids can also be natural.

\section{Dynamic Logic of Information Change}

Having introduced our explicit/implicit contrast for two well-known logics, we now move to other areas and see where it leads. Recall that on the semantic view taken so far, inquiry lies at the heart of both epistemic and intuitionistic logic. Clearly, knowledge and information do not function in isolation, but in an ongoing dynamic process of informational action, or in a social setting, interaction between agents. This process has been a recent focus of attention in logic design.

Statics and Dynamics Key informational actions that guide agents come in three kinds that work together in many scenarios. Inferences matter - but equally important are acts of observation and communication. Such acts, or events that embody them, are studied in dynamic-epistemic logics, by adding vocabulary for core actions to classical logics, and then analyzing the laws of knowledge change, [13].

Model Update Here is a system making the dynamic actions behind basic epistemic logic explicit by representing informational action as model change. The simplest case of such a change occurs with a public announcement or a similar public event $! \varphi$ that produces hard information, where one learns with total reliability that $\varphi$ is the case. This eliminates all worlds in the current model where $\varphi$ is false:

\footnotetext{
${ }^{6}$ One way of seeing finer differences here is in terms of computational complexity. Theories that are equivalent under translation, perhaps an inefficient one, may have different computational properties. We will not pursue this angle here, but complexity is a natural way of driving a finer wedge.

${ }^{7}$ Also, translations only work with the right deductive power on both sides. Axioms as in S5 or S4 finetune deductive power, but also signal a conceptual switch. The reflexive transitive accessibility relation of $S 4$ does not encode an $S 5$-style epistemic range, but a process of inquiry into the actual world, where non-persistent atoms model non-intuitionistic information retraction or world change.
} 


\section{from $M$}
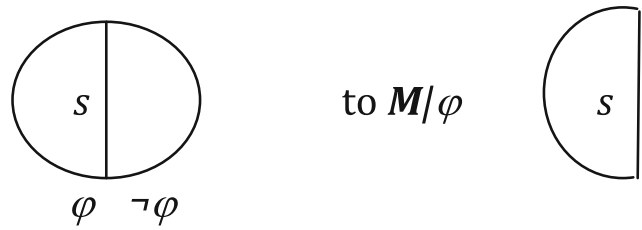

As we said when motivating epistemic models, getting information by shrinking a range of options is a common idea in many disciplines, that works for information flow by being told or through observation. This is often called hard information because of its irrevocable character: the update step eliminates all counter-examples.

Public Announcement Logic Public announcements are studied in $P A L$, a system that extends epistemic logic with a dynamic modality for truthful announcements:

$$
\boldsymbol{M}, s \mid=[! \varphi] \psi \quad \text { iff } \quad \text { if } \boldsymbol{M}, s \mid=\varphi \text {, then } \boldsymbol{M}|\varphi, s|=\psi
$$

This dynamic modality has a complete logic that can analyze delicate phenomena, such as complex epistemic assertions, say of current ignorance, changing truth value under update. This typically shows in order dependence: a sequence $! \neg K p ; ! p$ makes sense, but $! p ; ! \neg K p$ is contradictory. Here we only display the 'recursion law' for knowledge after update, which is the basic dynamic equation of hard information:

$$
[! \varphi] K \psi \leftrightarrow(\varphi \rightarrow K(\varphi \rightarrow[! \varphi] \psi))
$$

Together with the S5-laws for epistemic logic plus simple axioms for Boolean compounds after update this gives a complete axiomatization for $P A L$. Another interesting law demonstrating the dynamics of $P A L$ governs iterated updates:

$$
[! \varphi][! \psi] \chi \leftrightarrow[!(\varphi \wedge[! \varphi] \psi)] \chi
$$

Recursion axioms reduce formulas with dynamic operators to static base formulas, so the extension of our classical base logic is conservative in the usual explicit style.

General Dynamics There is a method here. One 'dynamifies' a given static logic, making its underlying actions explicit and defining them as model transformations. The heart of the dynamic logic is then a compositional analysis of post-conditions for the key actions via recursion laws. This leads to conservative extensions of the base logic, though some systems force redesign of their base, while some recent semantics no longer support all-out reduction. Many further notions can be treated in this style, such as changes in beliefs, inferences, issues, or preferences - by changing the ordering of worlds rather than eliminating them. Dynamic-epistemic logics also deal with public and private events in multi-agent scenarios such as games. ${ }^{8}$

\footnotetext{
${ }^{8}$ Even where a dynamic logic conservatively extends a base logic it may still affect our view of the statics. Consider the many modal logics with axioms matching conditions on accessibility relations. One can often analyze such conditions in dynamic terms. Say, transitive relations arise from an act $\boldsymbol{t c}$ of transitive closure: 'reflection' or 'exploration'. But then a $K 4$-modality $K \varphi$ is an ordinary modality $\square$ over models resulting from this action, making it a compound $[t c] \square \varphi$. This faithfully embeds $K 4$ over transitive models into propositional dynamic logic over arbitrary models. Thus, variety of of static modal logics may dissolve in favor of one base logic plus modalities for suitable model changes.
} 


\section{Implicit Dynamics in Intuitionistic Logic}

We have now extended epistemic logic, an explicit approach to knowledge, to a dynamic logic with explicit informational actions. Is there an implicit counterpart? Given our earlier discussion, it makes sense to search in the realm of intuitionism. We could just add the actions of PAL to intuitionistic logic, [4]. But can we be more implicit about informational actions, without putting them into explicit syntax?

Locating the Hidden Actions Intuitionistic models represent a process of inquiry, with endpoints as final stages where we know the truth about all proposition letters. What are the implicit steps in the background of such a process taking us from node to node? Moves from one state to a successor come in two kinds.

Example The hidden dynamics of intuitionistic models.

Consider two models $\boldsymbol{M}_{1}, \boldsymbol{M}_{2}$, where the first refutes the classical double negation law $\neg \neg p \rightarrow p$, and the second the law of 'weak excluded middle' $\neg p \vee \neg \neg p$ :

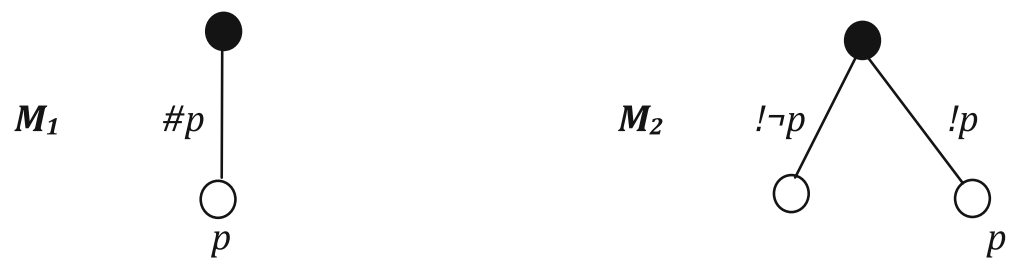

The annotations say that the two branches of $\boldsymbol{M}_{2}$ may be viewed as public announcements of which endpoints, viewed as classical valuations, the process can get to. This is like $P A L$-style learning by elimination of worlds. But in other intuitionistic steps, like the one in $\boldsymbol{M}_{1}$, there is no elimination, and we just get more proposition letters true at the next stage. One might view this move as a new kind of implicit informational action, namely, 'awareness raising' $\# \varphi$ that some fact $\varphi$ is the case, where awareness involves syntactic in addition to semantic information. ${ }^{9}$

Factual and Procedural Information But there is more than mere transposing of concerns from dynamic-epistemic logic. The tree structure of intuitionistic models registers two notions of semantic information on a par, a distinction also found in epistemic inquiry with long-term scenarios in learning theory, [36]:

(a) factual information about how the world is,

(b) procedural information about our current investigative process.

How we can get knowledge matters, not just what is the case. While endpoints record eventual factual information states, the branching tree structure of intuitio-

\footnotetext{
${ }^{9}$ With such a new operator, one could also make more general distinctions between 'aware' and 'unaware' versions of logical constants, say, implications - but this line seems unexplored so far.
} 
nistic models, both its available and its missing intermediate stages, encodes further non-trivial information: viz. agents' knowledge about the process of inquiry.

This challenges uniform views of how intuitionistic and epistemic logic connect. The epistemic logic for semantic information is $S 5$, while the Gödel translation into $S 4$ reflects a view of intuitionistic models as temporal processes of inquiry. Thus, an explicit counterpart to intuitionistic logic needs a temporal version of dynamicepistemic logic (cf. Footnote 5). Indeed, temporal 'protocol models' with designated admissible histories satisfying constraints on inquiry, [13], model procedural information in long-term processes of inquiry or learning beyond local dynamic steps.

Thus, both epistemic logic and intuitionistic logic have dynamic extensions having to do with inquiry, and these can be developed in both explicit and implicit styles. Moreover, this process is not routine and interesting new issues come to the fore.

Dynamic Semantics, Meaning as Information Change Potential Intuitionistic logic is not the only vehicle for a meaningful comparison with $P A L$. Explicit logics need not have unique implicit companions, there may be more matchings. Indeed, the more striking implicit counterpart to dynamic epistemic logics may well be another logical paradigm, that we will discuss now, raising new issues.

Here is a fundamental idea from dynamic semantics of natural language, going back to classical sources like $[31,56]$. The guiding intuition of this approach to language involves communication-oriented 'information change potential':

The meaning of an expression is its potential for changing information states of someone who accepts the information conveyed by the expression.

This sounds like a plea for taking informational actions seriously, as we did in the preceding section. But this time, they are treated, not by adding new operators, but implicitly, by loading the meanings of classical vocabulary with dynamic features.

Dynamic semantics comes in many forms. In what follows, we use Veltman's update semantics $U S$ for a modal propositional language and its novel account of consequence, [56], for a comparison with the explicit $P A L$ approach. In this semantics, on a universe of information states (in the simplest version, sets of atomic valuations representing ways the actual world might be), each modal propositional formula $\varphi$ denotes a state transformation $[[\varphi]]$ by the following recursion:

$$
\begin{aligned}
& {[[p]](S)=S \cap[[p]]} \\
& {[[\neg \varphi]](S)=S-[[\varphi]](S)} \\
& {[[\varphi \vee \psi]](S)=[[\varphi]](S) \cup[[\psi]](S)} \\
& {[[\varphi \wedge \psi]](S)=[[\psi]]([[\varphi]](S))} \\
& {[[\diamond \varphi]](S)=S, \text { if }[[\varphi]](S) \neq \emptyset, \text { and } \emptyset, \text { otherwise. }}
\end{aligned}
$$

Conjunction now stands for a dynamic notion: sequential composition of actions, while an existential modality becomes a 'test' on the current information state.

As with intuitionistic logic, these new meanings for old operators result in deviations from classical logic. In particular, conjunction is no longer commutative, reflecting the typical order dependence of dynamic acts. Facts about the informational process are now encoded in the logic of the logical operators in this system. 
This encoding becomes even more pronounced with the introduction of a new notion of dynamic consequence saying that, after processing the information in the successive premises, the conclusion has no further effect:

$\varphi_{1}, \ldots, \varphi_{n} \mid=\psi$ iff for every information state $X$ in any model, $\varphi_{n}\left(\ldots\left(\varphi_{1}(X)\right)\right)$

is a fixed point for $[[\psi]]$ : i.e., this set stays the same under an update $[[\psi]]$.

Dynamic consequence differs from classical consequence, and its deviations encode typical features of the update process, such as sensitivity to order or multiplicity of premises. But equally typically for the implicit style, what changes here are the classical laws of logic, not its methodology. Completeness theorems exist for dynamic consequence.

There are many sophisticated systems of dynamic semantics for other classes of expressions, with different notions of meaning, state change and dynamic consequence, cf. [28] - but the above seems a fair introduction to one basic mechanics.

\section{Dynamic Semantics Versus Dynamic Logic of Information}

PAL and Dynamic Semantics Dynamic epistemic logics like PAL and update semantics for propositional logic both take information change seriously, with analogous scenarios and intuitions. And both systems have a precise account for the dynamics of informational actions. But one does so explicitly, and the other implicitly:

Dynamic semantics keeps the actions implicit, while giving the old language of propositional logic richer dynamic meanings supporting a new notion of consequence, with a theory that differs from standard propositional logic.

Dynamic epistemic logic makes the actions explicit, provides them with explicit recursion laws, extends the old base language while retaining the old meanings for it, and in all this, it still works with standard consequence.

Again, there are some interesting issues here, as with the case of epistemic and intuitionistic logic, concerning our choice of a semantic platform for making the comparison. For instance, epistemic models look more like third-person descriptions of multi-agent informational situations, while the usual models for dynamic semantics look more like first-person internal perspectives of those agents. We will not pursue this issue here, but it will be discussed further in Section 15 .

As before, this is not just a matter of attaching two labels 'implicit' and 'explicit'. Seeing things in terms of our contrast leads to new questions and open problems. One straightforward illustration concerns new system design.

Inquiry and Questions A current innovation in dynamic semantics is inquisitive semantics for natural language, [20], where formulas get richer 'inquisitive meanings' reflecting their role in, not just conveying information, but also in directing discourse. The resulting logic is a non-classical intermediate logic related to Medvedev's logic of problems from the intuitionistic tradition. Our analysis then suggests 
the design of an explicit counterpart. Such dynamic-epistemic logics of inquiry - not tied to natural language, but closer to epistemology and learning theory - exist, and they involve explicit acts of 'issue management', where questions and related actions modify current issue structures on top of epistemic models, and the logical language has explicit modalities for such model-changing actions, [16, 33].

In the remainder of this section, we go into more depth on the foundational issue of how the two views of dynamics are related, and point out new issues that emerge.

Translations Between US and S5 As with epistemic and intuitionistic logic, there are translations between dynamic semantics and dynamic-epistemic logic, but they involve new issues. Our first observation comes from [11]:

Fact There is a faithful translation from update-validity into the modal logic S5.

The following is a recursive map $\operatorname{tr}$ from propositional formulas $\varphi$ to modal formulas $\operatorname{tr}(\varphi)(q)$, where $q$ is a fresh proposition letter (note the clause for conjunction):

$$
\begin{array}{ll}
\operatorname{tr}(p) & =q \wedge p \\
\operatorname{tr}(\neg \varphi) & =q \wedge \neg \operatorname{tr}(\varphi) \\
\operatorname{tr}(\varphi \vee \psi) & =\operatorname{tr}(\varphi) \vee \operatorname{tr}(\psi) \\
\operatorname{tr}(\diamond \varphi) & =q \wedge \diamond(q \wedge \operatorname{tr}(\varphi)) \\
\operatorname{tr}(\varphi \wedge \psi) & =[\operatorname{tr}(\varphi) / q] \operatorname{tr}(\psi)
\end{array}
$$

Now ${ }^{10}$ consider $S 5$-models $M(q:=S)$ marked to show that $q$ denotes the set of worlds $S$. The $U S$ semantics works here on sets of worlds $X$ to produce values $[[\varphi]]^{\boldsymbol{M}}(S)$. Then the following equivalence holds for all $U S$-formulas $\varphi$ and subsets $S$ of $\boldsymbol{M}$ :

$$
\boldsymbol{M}(q:=S), s \mid=\operatorname{tr}(\varphi) \quad \text { iff } \quad s \in[[\varphi]](S)
$$

As a corollary, ${ }^{11}$ for update validity, we have that

$$
\varphi_{1}, \ldots, \varphi_{n} \mid=_{U S} \psi \text { iff } \mid=S 5 \operatorname{tr}\left(\varphi_{1} \wedge \ldots \wedge \varphi_{n} \wedge \psi\right) \leftrightarrow \operatorname{tr}\left(\varphi_{1} \wedge \ldots \wedge \varphi_{n}\right)
$$

In fact, connections run both ways. There is also a converse embedding:

Fact There is a faithful translation from $S 5$-validity into update validity.

To see this, transform $S 5$-formulas $\varphi$ into their normal form $n f(\varphi)$ of modal depth 1. Then, for $S 5$-validities, the update function $[[n f(\varphi)]]$ is the identity on all sets, while for non-validities, on any counter-model, $[[n f(\varphi)]]$ returns the empty set.

System Identity Now an earlier issue returns. Do the preceding results say that US is the same system as $S 5$ ? Our translations reduce valid consequence both ways, which is enough for the standard notion of system equivalence. But the intuitive novelty of $U S$ is that it does something more: it can express the dynamics of model change. To

\footnotetext{
${ }^{10}$ We may have overdosed on occurrences of ' $q \wedge$ ' here, but this makes proofs more perspicuous.

${ }^{11}$ Here is the crucial inductive step. $\boldsymbol{M}(q:=S), s=\operatorname{tr}(\varphi \wedge \psi)$ iff $\boldsymbol{M}(q:=S), s=[\operatorname{tr}(\varphi) / q] \operatorname{tr}(\psi)$ iff (using an obvious substitution lemma, with [] ordinary denotation brackets) $\boldsymbol{M}\left(q:=[\operatorname{tr}(\varphi)]^{\boldsymbol{M}(q:=S)}\right), s=\operatorname{tr}(\psi)$ iff (by the inductive hypothesis) $\boldsymbol{M}(q:=[[\varphi]](S)), s \models \operatorname{tr}(\psi)$ iff $s \in[[\psi]]([[\varphi]](S))$ iff $s \in[[\varphi \wedge \psi]](S)$.
} 
some extent, the details of our first translation give information about this aspect, too: $S 5$ can define model changes in ambient sets $q$ using the formulas $\operatorname{tr}(\varphi)$ as indicated, and this process even simulates the working of $U S$ in a recursive manner. And yet the two systems feel intensionally different, and US seems a new discovery.

I must leave the matter of detecting finer intensional differences open here, but will return to the issue of adequately comparing dynamic components by drawing in the logic $P A L$.

PAL and S5 Similar points can be made concerning public announcement logic.

Fact There are faithful translations between $P A L$-validity and modal S5.

This time, the reason is that the recursion laws provide an effective truth-preserving translation from all $P A L$-formulas with dynamic modalities into the $S 5$ base language, while for that static fragment, $P A L$ is a conservative extension of $S 5$.

Comparing US and PAL Directly Composing their mutual translations with S5 gives faithful embeddings between $U S$ and $P A L$, our paradigms of implicit and explicit dynamics. But despite what was said before, going via the static logic $S 5$ does not relate the dynamic character of both approaches directly. Can we do better?

There is an obstacle here. Update semantics typically recurses on the structure of modal propositional formulas interpreted as updates, whereas the characteristic axioms of PAL do not recurse on the inner structure of announcements $! \varphi$, but on the postconditions $\psi$ for the dynamic modalities $[! \varphi] \psi$. To overcome this difference, we merge, and enrich $P A L$ with conversational programs built from actions $! \varphi$ by standard program operations of union and sequential composition, [13]. The following translation then arises, where $T$ stands for the logical expression 'True':

$$
\begin{aligned}
\operatorname{Tr}(p) & =! p \\
\operatorname{Tr}(\neg \varphi) & =! \neg<\operatorname{Tr}(\varphi)>T \\
\operatorname{Tr}(\varphi \vee \psi) & =\operatorname{Tr}(\varphi) \cup \operatorname{Tr}(\psi) \\
\operatorname{Tr}(\diamond \varphi) & =! \diamond<\operatorname{Tr}(\varphi)>T \\
\operatorname{Tr}(\varphi \wedge \psi) & =\operatorname{Tr}(\varphi) ; \operatorname{Tr}(\psi)
\end{aligned}
$$

Now it is easy to show that, for models $\boldsymbol{M}$ whose domain is the set $S$ that:

$$
[[\varphi]](S)=\{s \in S|\boldsymbol{M},|=<\operatorname{Tr}(\varphi)>\mathrm{T}\}
$$

To see how this works, compare the $P A L$ program $! \diamond \neg<! p>T ; ! p$ for the consistent $U S$ formula $\diamond \neg p \wedge p$ with the program $! p ; ! \diamond \neg<! p>T$ for the inconsistent $U S$ formula $p \wedge \diamond \neg p$.

$\operatorname{Tr}$ does not translate $U S$ updates into single $P A L$ actions, but it comes close. Earlier on, we saw how public announcements are closed under sequential composition, and hence $\operatorname{Tr}(\varphi \wedge \psi)$ amounts to announcing just one suitable S5-formula. ${ }^{12}$

\footnotetext{
${ }^{12}$ Our result is just a pilot case, and much more can be done. E.g., [23] translates dynamic predicate logic, [31], another well-known dynamic semantics format, into standard dynamic logic of programs. One can also give translations from inquisitive logics into logics of issue management, and so on.
} 
Open problem Is there also a direct translation from $P A L$ actions into US updates?

Discussion These translations again have various uses. Decidability of dynamic consequence follows from that for $S 5$. And we can use results about $P A L$ as road signs for US. E.g., the logic of $P A L$ extended with conversational programs that allow finite iterations is non-axiomatizable and not arithmetically definable, [43]. So, dynamic semantics for discourse rather than sentences might run the same complexity risk.

But earlier reservations apply: despite the translations, $U S$ and $P A L$ seem intuitively different. For instance, recall our notion of 'procedural information' in intuitionistic models. Viewing PAL as a logic of inquiry, a generalized semantics of 'protocol models' with restricted temporal histories of updates makes sense, [13]. This natural modification changes the laws of $P A L$, and it blocks the translation of $P A L$ into $S 5$. However, it is unclear if protocol models make sense in dynamic semantics. Also, $P A L$ update has a natural extension to dynamic-epistemic logics with much more drastic model changes modeling the dynamics of partly private information, and it is unclear if these richer transformations have any role in a dynamic semantics.

What this discussion suggests is a more demanding criterion of system identity: equality or difference in 'natural generalizations'. But is there a formal basis to this, or would the criterion merely concern our current powers of imagination?

We found natural translations between dynamic semantics and dynamic-epistemic logics. Still, implicit and explicit approaches do not collapse, and again we might be content with creating merges. Either way, the realm of information dynamics seems a rich source for our explicit/implicit contrast, raising interesting issues of its own.

\section{Dynamic Logics of Soft Information}

Our discussion so far centered on the statics and dynamics of knowledge. However, the implicit/explicit contrast applies just as well to logics of belief, perhaps the more important attitude in agency. The case of belief shows interesting new features and suggests new comparisons between implicit and explicit logic systems. We start with belief dynamics in explicit style, moving to implicit counterparts later.

Belief and Conditional Belief Epistemic-doxastic models for belief order the earlier bare epistemic ranges by a relation of 'relative plausibility' $\leq x y$ between worlds $x, y$. These models interpret operators of absolute and conditional belief:

$$
\begin{aligned}
& \boldsymbol{M}, s=B \varphi \quad \text { iff } \quad \boldsymbol{M}, t=\varphi \text { for all } t \sim s \text { maximal in the order } \leq \text { on }\{u \mid u \sim s\} \\
& \left.\boldsymbol{M}, s \mid=B^{\psi} \varphi \text { iff } \quad \boldsymbol{M}, t=\varphi \text { for all } \leq \text {-maximal } t \text { in }\{u \mid s \sim u\} \text { and } \boldsymbol{M}, u \mid=\psi\right\}
\end{aligned}
$$

But there is a richer repertoire of epistemic notions available on this models. For instance, on binary world-independent orderings, a good addition is 'safe belief', a standard modality intermediate in strength between knowledge and belief:

$$
\boldsymbol{M}, s \mid=[\leq] \varphi \quad \text { iff } \quad \boldsymbol{M}, t=\varphi \text { for all } t \text { with } s \leq t
$$


Logics for conditional belief are like those of $[19,41]$. For a more general picture of natural modalities that can be defined on these models, see [5].

Belief Change Under Hard Information Beliefs guide our decisions and actions, going beyond what we know. But beliefs can be wrong, and new information can lead to correction and learning. One trigger for belief revision are the earlier public announcements. Here is the recursion law governing the matching model changes:

$$
[! \varphi] B \psi \leftrightarrow\left(\varphi \rightarrow B^{\varphi}[! \varphi] \psi\right)
$$

A similar principle for updating conditional beliefs axiomatizes the system completely. There is also a recursion law for safe belief under public announcement, which is even simpler. The following equivalence holds on plausibility models:

$$
[! \varphi][\leq] \psi \leftrightarrow(\varphi \rightarrow[\leq][! \varphi] \psi)
$$

Belief Change Under Soft Information But richer belief models also support new transformations. In addition to hard information, there is soft information, when we take a signal as serious, but not infallible. Its mechanism is not eliminating worlds, but changing plausibility order. A widely used soft update is 'radical upgrade':

$\Uparrow \varphi$ changes a current model $\boldsymbol{M}$ to $\boldsymbol{M} \Uparrow \varphi$, where all $\varphi$-worlds become better than all $\neg \varphi$-worlds; within these zones, the old order remains.

The dynamic modality for radical upgrade is interpreted as follows:

$$
\boldsymbol{M}, s \mid=[\Uparrow \varphi] \psi \text { iff } \boldsymbol{M} \Uparrow \varphi, s \mid=\psi
$$

and its dynamic logic can again be axiomatized completely using recursion laws.

Logics of Belief Change Recursion laws exist for belief changes under a wide variety of soft events representing different levels of trust or acceptance for new information, [5, 28]. An area where this variety makes special sense is Learning Theory, [27]: different update rules induce different policies for reaching true belief in the limit. The Handbook [21] has details on the landscape of modal logics for belief change, plus connections with $A G M$-style postulational approaches.

The systems presented here are explicit in a double sense. Not only do events and acts that usually stay in the background of logical systems become first-class objects of study, but also, dynamic logics for knowledge and belief have explicit syntax and laws for these actions. The new structure is not put into the meanings of the original language, and so we get conservative extensions of earlier static logics, although sometimes there is a need for some redesign of the original static vocabulary.

\section{Non-Monotonic Consequence Relations as Implicit Devices}

Next, how can we do belief revision in an alternative implicit style? One line runs via dynamic semantics, with new meanings for linguistic expressions such as epistemic modals, [49, 56, 57]. All our earlier points apply, but we will not pursue them here. 
Instead, we show how our contrast can also take us, perhaps surprisingly, to an area of implicit logic that seems quite different from those discussed so far.

Varieties of Consequence In the 1980s, the study of different consequence relations in common sense problem solving started in Artificial Intelligence, and it has since entered other fields. In particular, the notion of circumscription [42, 53] says that, in problem solving or related tasks, the following inferences are allowed:

A conclusion need not be true in all models of the premises, but only in the most preferred, or most plausible models.

Thus, problem solving involves only inspection of currently most relevant cases.

This style of reasoning deviates from classical logic. In particular, it is 'non-monotonic': a conclusion $C$ may follow from a premise $P$ in this sense, but it may fail to follow from the extended set of premises $P, Q$. For, the maximal models within the set of models for the conjunction $P \wedge Q$ need not be maximal among the models of $P$.

Many forms of defeasible inference have been studied, given the large repertoire of human reasoning styles - and complete structural rules or proof systems have been found, following what Bolzano and Peirce already advocated in the $19^{\text {th }}$ century. These systems, that usually drop some classical rules, while retaining modified variants, are typically taken to encode basic features of such styles of reasoning.

Non-Standard Consequence as Implicit Logic This modus operandi is highly reminiscent of an implicit approach in our sense. What is new about the reasoning practice under study is not explicitly on the table, but it shows in differences and analogies with standard properties of classical consequence for a classical language.

Making it Explicit But non-standard consequence relations have concrete motivations, they do not just arise by tinkering with classical structural rules. So, can we provide alternative explicit accounts leaving the notion of consequence standard, while adding vocabulary to bring out the origins of the new consequence notions? As in all our earlier illustrations, we need a semantic platform for doing so, and the choice for this will depend on the concrete motivation for the new consequence relation. The following case study highlights the role of belief in circumscription though explicitizing consequence relations may well involve other notions, too.

From Inference to Belief Change Revisiting the original AI scenarios, one can also construe problem solving differently. We have beliefs about a problem and where a solution might go, based on scenarios that seem most plausible to consider either deep beliefs based on experience in problem solving, or light beliefs as lacking considerations to the contrary. Then, as we take in new information relevant to the problem, this set of scenarios changes, and beliefs are modified. ${ }^{13}$ Now this fits precisely with our models of beliefs. For instance, a circumscriptive consequence

$$
\varphi_{1}, \ldots, \varphi_{n} \Rightarrow \psi
$$

\footnotetext{
${ }^{13}$ An early source for this style of thinking in computer science is Levesque's logic of belief, [40].
} 
translates into our earlier dynamic logic for belief change, using the formula

$$
\left[! \varphi_{1}\right] \ldots\left[! \varphi_{n}\right] B \psi
$$

This translation explains the deviations of non-monotonic logic from classical consequence, as the structural rules of circumscription now follow from the dynamic logic of belief revision. For instance, it is easy to see how $[! \varphi] B \psi$ does not imply $[! \varphi][! \alpha] B \psi$ for all formulas $\alpha$ - except for special cases. This explanation of the deviant inferential behavior has two sources: the key attitude in practical reasoning is fallible belief rather than knowledge, and also, we have explicit dynamic events. ${ }^{14}$

Remark There are well-known analogies between non-monotonic consequence and conditionals in the style of Lewis [38]. Instead of [! $\varphi] B \psi$, this might favor conditional belief $B^{\psi} \varphi$ pre-encoding effects of learning that $\psi$. The two versions are not quite the same, as update $! \psi$ restricts a model to its $\psi$-worlds, while a conditional can still look at $\neg \psi$-worlds when evaluating $\varphi$. But these details need not concern us here.

Either way, our general points apply. The juxtaposition of perspectives raises interesting issues. Again we see a trade-off between implicit and explicit approaches:

nonstandard consequence classical language, deviant rules of reasoning explicit dynamic reanalysis new language with belief and action modalities, consequence is just classical consequence.

On the second approach, non-standard ${ }^{15}$ reasoning is a mixture of classical reasoning and further features of informational actions, not a family of radical alternatives. Dynamic logics of belief change enrich the original language with informational events and attitude changes, but then work conservatively with a classical consequence relation, explaining deviant features of non-standard consequence by attitude and information change through the recursion laws for the new dynamic operators. In the following section, we evaluate this difference in approaches.

\section{Comparisons and Switches}

We have seen that non-monotonic consequence relations can be translated faithfully into a classical logic with operators for attitudes and informational events. But as before, this does not identify the two perspectives: one can still have advantages over the other. For instance, implicit approaches focus on structural rules, which are a natural high-level vantage point allowing for elegant theory. On the other hand, an explicit dynamic approach provides an emancipation of informational events in problem solving that is of interest per se, as it adds new events beyond inference.

\footnotetext{
${ }^{14}$ This very simple analysis is not the only explicit view of belief and non-monotonic logic. E.g., on the richer epistemic view in [52], default reasoning submits candidates for belief while further actions then sift available evidence that supports one's eventual considered beliefs.

${ }^{15}$ It should be noted that non-standard consequence relations, too, can support the introduction of new vocabulary beyond the classical language, for reasons discussed in Sections 4 and 15.
} 
New Dynamic Logics A neutral two-way view here sees switching perspectives, [13]. In one direction, given an implicit non-standard notion of consequence, one can tease out informational or other events motivating it, and write their explicit dynamic logic. This style of analysis, backed up by mathematical representation theorems, replaces non-standard deviation from classical logic into dynamic extension of classical logic. Explicit events behind non-standard notions of consequence are sometimes easy to find, as in the above analysis of circumscription, but there is no automatic method for this art - and there are unresolved challenges concerning major substructural logics based on proof-theoretic resource intuitions, [48]. ${ }^{16}$

New Notions of Consequence Vice versa, given an explicit dynamic logic of informational events, one can package some basic structure in the form of new consequence relations, and study those per se. The latter move can even add to our fund of styles of reasoning. Here is an illustration. Logics of belief change predict the existence of new styles of inference based on their repertoire of different informational events and attitudes. In particular, problem solving may involve different attitudes, such as both knowledge and belief, and also, it may take some new information as hard, and some in the earlier soft sense, leading to variants of circumscription such as

$$
\begin{array}{ll}
\text { soft-weak circumscription } & {\left[\Uparrow \varphi_{1}\right] \ldots\left[\Uparrow \varphi_{n}\right] B \psi} \\
\text { soft-strong circumscription } & {\left[\Uparrow \varphi_{1}\right] \ldots\left[\Uparrow \varphi_{n}\right] K \psi}
\end{array}
$$

where the premises are just taken as soft upgrades, not as public announcements. Different structural rules will then encode differences in the underlying process of drawing a conclusion. Thus, we generate new notions of consequence, and even more would arise with other mixtures of knowledge, belief and update actions.

Thus, in the study of consequence relations, implicit and explicit approaches live in harmony, and we can often perform a Gestalt switch from one to the other. Such switches also suggest precise mathematical system translations in our earlier sense.

Philosophical Repercussions While the preceding analysis may seem mainly technical, well-known positions based on non-standard logics come under pressure by an explicit-style reanalysis. Existence of different consequence relations on a par has led to the thesis of Logical Pluralism, a view that logic should acknowledge competing views on the nature of logical consequence, and perhaps also other core notions of the field, [10]. But in our view, this grand conclusion depends on taking the implicit methodology for granted. On a dynamic explicit reanalysis as presented here, the competition between consequence relations disappears, and we get compatible extensions of classical logic without any commitment to competition. The

\footnotetext{
${ }^{16}$ Once substructural logics have a semantics, however, our style of analysis applies. Consider the wellknown models for resource logics such as relevant or linear logic in terms of information pieces that can be combined by a binary operation of merging, [48]. Now we can introduce an explicit modality $<+>\varphi \psi$ true at merges of $\varphi$-points and $\psi$-points, and translate substructural systems into classical modal logics with this additional binary modality and further explicit companion operators. A special case of this kind of system will be used in the analysis of truth maker logic in Section 12.
} 
second view need not be superior to the first, but its very existence undermines strong conclusions arising from looking at consequence in only one stance. ${ }^{17}$

\section{Further Examples}

We have seen how the implicit/explicit contrast runs through both static and dynamic logics for knowledge and belief, as well as for logics for consequence relations. Further examples in this epistemic line can be found by moving from information flow to design stances in information-driven agency and games: for instance, in [14], implicit logic games and explicit game logics are naturally entangled strands throughout. But once one sees the contrast put forward in this paper, it applies to any part of logic whatsoever, not just information and agency. To demonstrate this broader range for our analysis, we discuss two examples, one taken from the philosophy of physics and one from contemporary metaphysics. Again, these topics raise new issues of their own that we will only touch upon in what follows.

Quantum Logic Our first example concerns a stronghold of non-classical logic since the 1930s. Consider the field of quantum logic, where the classical distributive law

$$
(p \wedge(q \vee r)) \leftrightarrow((p \wedge q) \vee(p \wedge r))
$$

fails in the domain of physical quantum phenomena. There are of course reasons for this failure: measurements disturb the current state of a physical system - but this is left implicit in quantum logic. There is a long tradition of research in this area, which has resulted in an extensive algebraic and modal theory of quantum logics.

The first explicit companion to all this seems the dynamic measurement logic of Baltag and Smets, cf. [6]. Their system of Quantum PDL has dynamic modalities for measurement actions that satisfy perspicuous laws mirroring physical quantum facts, but it remains squarely based on classical logic. In doing so, it explains all the deviant features of quantum logic in a uniform manner as properties of a small fragment of the explicit language. For instance, failure of distributivity becomes failure of actions to distribute over choice, a well-known phenomenon in logics of computation, which has nothing to do with propositional logic. But an explicit dynamic logic of measurement can also express further significant properties of physical systems, and analyze more types of measurement action on these, making traditional quantum logic a poor projection of what goes on from a physical point of view. ${ }^{18}$

Discussion This is not just reformulation into an explicit format: again, there are serious philosophical consequences. Quantum mechanics was famously touted by Quine, [47], and Putnam, [46], as a realm where not even the basic laws of logic are immune

\footnotetext{
${ }^{17}$ As pointed out by a referee, this need not mean that pluralism goes away. On the analysis presented here, pluralism for consequence relations might now morph into pluralism for different natural extensions of the vocabulary of classical logic. But in this version, pluralism is much less striking.

${ }^{18}$ An additional virtue is the analogy with our earlier dynamic-epistemic logics of knowledge change, making it possible to add epistemic considerations of agency to quantum information theory, [7].
} 
to revision in scientific theory construction. Taken for granted in this analysis was that quantum logic in implicit style is the only game in town. But this claim dissolves when we have a mathematically elegant and conceptually perspicuous classical logic that explicitly puts measurement where it belongs: at center stage.

This brief exposition may not do justice to explicit dynamic quantum logic, but suffice it to say that this new approach placing measurement actions and quantum information flow at center stage is more than just logic-internal system redesign. It fits well with a substantive topic, viz. recent investigations into analogies between the foundations of quantum mechanics and theories of computation.

Truth Maker Semantics Our second example shows our contrast at work in a very recent development. 'Truth maker semantics' has been touted as a hyper-intensional paradigm springing the bounds of standard modal logic, cf. [25]. In our terms, truth making is an implicit approach changing the meanings of the logical constants to reflect metaphysically (or, in other applications, epistemically) important structure, and defining new notions of consequence based on these changed meanings. Thus, it makes sense to look for a translation from truth maker logic into an explicit companion, namely, a standard modal logic over the same class of models.

We give a brief explanation of how this can be done for one simple pilot system.

Models for truth making $\boldsymbol{M}$ are tuples $(S, \leq, V)$ with objects $s$ in $S$ viewed as parts of the world or abstract states. The binary relation $\leq$ is a partial order between states. The relation of supremum $s=\sup (t, u)$ (lowest upper bound) says that object $s$ is a sum or merge of the $t$ and $u$. It is often assumed in the literature that all suprema exist, often as 'impossible worlds' in case the merged states are incompatible.

The simplest relevant language here is a propositional logic with connectives $\neg$, $\wedge, \vee$. For atomic $p$, a valuation $V$ records which states in $S$ make $p$ true, the set $V^{+}(p)$, or false, $V^{-}(p)$. This can be made subject to further constraints: for instance, that no state makes a proposition both true and false. The truth definition is as follows:

$$
\begin{array}{lll}
\boldsymbol{M}, s \mid=p & \text { iff } & s \in V^{+}(p) \\
\boldsymbol{M}, s=\mid p & \text { iff } & s \in V^{-}(p) \\
\boldsymbol{M}, s \mid=\neg \varphi & \text { iff } & \boldsymbol{M}, s=\mid \varphi \\
\boldsymbol{M}, s=\neg \varphi & \text { iff } & \boldsymbol{M}, s=\varphi \\
\boldsymbol{M}, s \mid=\varphi \wedge \psi & \text { iff } & \text { there exist } t, u \text { with } s=\sup (t, u), \boldsymbol{M}, t \mid=\varphi \text { and } \boldsymbol{M}, u \mid=\psi \\
\boldsymbol{M}, s=\mid \varphi \wedge \psi & \text { iff } & \boldsymbol{M}, s=\mid \varphi \text { or } \boldsymbol{M}, s=\mid \psi \\
\boldsymbol{M}, s \mid=\varphi \vee \psi & \text { iff } & \boldsymbol{M}, s \mid=\varphi \text { or } \boldsymbol{M}, s \mid=\psi \\
\boldsymbol{M}, s=\mid \varphi \vee \psi & \text { iff } & \text { there exist } t, u \text { with } s=\sup (t, u), \boldsymbol{M}, t=\mid \varphi \text { and } \boldsymbol{M}, u=\mid \psi
\end{array}
$$

One can also define further notions of 'loose' and 'inexact' truth and false making. Next one can define various notions of consequence, such as each truth maker for all premises being a truth maker for the conclusion, or each truth maker of the premises being extendable to one for the conclusion, as well as versions that add conditions on false making. All support different laws for the propositional base language. Thus propositional logic is the locus for the new conceptual analysis. 
Modal Information Logic Now essentially these same structures have been around in modal logic since the 1990s as models of abstract information states, [11]. The universal modality $[\uparrow] \varphi$ describes upward structure from a point, and downward $[\downarrow] \varphi$ describes weaker information. The logic is then temporal $S 4$. Where suprema exist in the order, the logic describes them using binary modalities:

$$
\begin{array}{ll}
\boldsymbol{M}, s=<\sup >\varphi \psi & \text { iff } \quad \text { there exist } t, u \text { with } s=\sup (t, u), \boldsymbol{M}, t=\varphi \text { and } \boldsymbol{M}, u=\psi \\
\boldsymbol{M}, s \mid=<\inf >\varphi \psi & \text { iff } \quad \text { there exist } t, u \text { with } s=\inf (t, u), \boldsymbol{M}, t=\varphi \text { and } \boldsymbol{M}, u \mid=\psi
\end{array}
$$

It is easy to show that $<s u p>p q$ is not definable in the temporal modal language, making this a natural extension of the ordinary modal logic $S 4$.

As for laws of reasoning, the modal logic of information has interesting validities, but details are not relevant here. One principle that fails though is associativity:

$$
<\text { sup }><\text { sup }>\varphi \psi \alpha \rightarrow<\text { sup }>\varphi<\text { sup }>\psi \alpha
$$

The reason is that, unlike in truth maker semantics, we do not demand existence of all suprema in our partial orders. The modal logic of information structures is axiomatizable, but a major open problem is whether it is decidable, [12]. ${ }^{19}$

Translating Truth Maker Logic Into Modal Information Logic The models just described and their modal logic are an explicit companion to truth maker logic. And the connection is very close. Here is a two-component recipe for translating from the language of implicit truth maker logic into explicit modal logic, where the simultaneous use of variants + and - is a standard trick in reducing three-valued logic to classical logics.

Take new proposition letters $p^{+}$and $p^{-}$for each atomic proposition letter $p$. Now, for each propositional formula $\varphi$, we recursively extend this double set-up as follows, with clauses closely following the above truth definition:

$$
\begin{aligned}
& (\neg \varphi)^{+}=(\varphi)^{-} \quad(\neg \varphi)^{-}=(\varphi)^{+} \\
& (\varphi \wedge \psi)^{+}=<\sup >(\varphi)^{+}(\psi)^{+} \quad(\varphi \wedge \psi)^{-}=(\varphi)^{-} \vee(\psi)^{-} \\
& (\varphi \vee \psi)^{+}=(\varphi)^{+} \vee(\psi)^{+} \quad(\varphi \wedge \psi)^{-}=<\sup >(\varphi)^{-}(\psi)^{-}
\end{aligned}
$$

Theorem $\varphi_{1}, \ldots, \varphi_{n} \vDash \psi$ is valid in truth maker semantics iff the consequence $\left(\varphi_{1}\right)^{+}, \ldots,\left(\varphi_{n}\right)^{+} \vDash(\psi)^{+}$holds in modal information logic.

We do not provide a formal proof, but the translation is almost self-explanatory.

The translation can accommodate a notion of inexact truth making as $\langle\downarrow\rangle \varphi$, and other modal combinations can deal with 'loose truth making'. Adding strict versions $\left[\uparrow^{s}\right],\left[\downarrow^{s}\right]$ of the order modalities defines strict truth making as $\left[\downarrow^{s}\right] \neg \varphi \wedge \varphi \wedge\left[\uparrow^{s}\right] \neg \varphi$. Also, the earlier-mentioned varieties of consequence are easily seen to be modally

\footnotetext{
${ }^{19}$ Since logics with associative modalities often encode undecidable word problems, this might be a warning sign for the use of 'impossible worlds' as suprema in truth making. The practice of throwing in such worlds looks like harmless smoothening of the universe, but it induces associativity.
} 
definable, and with a little more effort, so are special conditions on for truth maker denotations such as closure under merge, or convexity.

Discussion What does our translation achieve? First, it brings methods from modal logic to the study of truth making - though not all issues are settled automatically, such as decidability or axiomatization. But the translation also has a clear philosophical point: truth maker logic is entirely compatible with classical modal logic, thus refuting claims about irreducible hyper-intensionality. Moreover, our translation illustrates a genre: similar reanalyses can be given of other proposed hyperintensional semantics. Finally, this is not just rephrasing. In exploring metaphysics, an explicit modal logic might be a serious rival to classical logic reinterpreted via truth making, as it puts no linguistic constraints on how to reflect metaphysical structure. If we truly love something, why not give it its own new vocabulary?

\section{Implicit and Explicit Stances at Work}

After this array of examples, it might seem time for a precise definition of the implicit/explicit contrast. But we do not have one to offer, and we doubt that a definition exists covering all cases in a useful manner. Even so, we did identify recognizable general features. Implicit approaches enrich old meanings, and locate important information in deviant notions of consequence - explicit approaches introduce new vocabulary, but conservatively extend classical logic. And with this difference comes plurality of alternative logics for implicit approaches, and plurality of extensions of classical logic on explicit approaches. These features should help recognize the two styles when one sees them at work in logical system design. Some further thoughts on limitations of our implicit-explicit analysis will be found in Section $15 .^{20}$

But our main concern in this paper is not assigning labels, and Scholastic taxonomy of existing systems. Our aim is much more activist. As we have shown by many examples, seeing the contrast raises interesting new issues, both practical and theoretical. We summarize a few strands that occurred in the preceding sections.

Finding Complementary Analyses If we see one stance on a topic, we can usually find a dual one. Thus our contrast becomes a force for logical system design. We saw this with dynamic semantics of questions, which suggested an explicit companion logic of issue modifying events. And conversely, explicit logics of belief change suggested new implicit notions of consequence in the area of non-monotonic logic.

Transfer of Ideas Different stances on the same thing facilitate creative borrowing, since their agendas may differ. For instance, epistemic logic has a rich tradition of multi-agent and group knowledge. Intuitionistic logic can then profit from the same ideas, creating accounts of mathematics closer to research as a social activity, cf. [2].

\footnotetext{
${ }^{20}$ Recall that our contrast applied to activities of design, and only in a derived sense to the systems produced. It may be hard to classify logics as implicit or explicit when we disregard their genesis.
} 
But one can also borrow ideas inside one stance. For instance, intuitionistic logic started from the proof-theoretic BHK interpretation of the logical constants, which met up with semantics only afterwards. Could a similar proof-theoretic analysis apply to dynamic semantics, a major implicit paradigm for information dynamics? Or, for another example inside the implicit realm: can BHK-style proof analysis be taken to non-monotonic logics, and thus to belief rather than knowledge?

Translation and Identity Criteria for Logics The explicit/implicit contrast also suggests new mathematical issues of translation or reduction between logical systems. We have given some new examples, and no doubt much more can be proved about translating between implicit and explicit logics. Even so, there is no automatic algorithm for turning one sort of system into the other. Finding illuminating counterparts as we have done is an art rather than a science, and it may well remain so.

We have also suggested that, even when implicit and explicit logics can be mutually embedded under translation - clearly a telling fact - subtle differences may remain. Here we encountered a general issue in the foundations of logic. There is no generally accepted criterion for when two (presentations of) a logical system should count as the same. Mutual interpretability is a significant notion of equivalence that allows for much transfer of information, so we should always see if it occurs, but it need not be the last word. ${ }^{21}$ In fact, one vexing problem that makes it hard to judge the merits of this notion is a scarcity of negative results. There are no general methods showing non-translatability between logical systems. Perhaps, in the end, there is too much translatability in the realm of logics, and a finer sieve is needed.

Merging Where we cannot translate different stances into each other, a weaker connection is compatibility in meaningful merges. Many systems in the literature combine implicit and explicit features: intuitionistic modal logics, [22], merged logic games and game logics, [14], dynamic-epistemic inquisitive logics, [50], joint lineartemporal logics, and so on. Often, these merges feel natural. A recent case is the intuitionistically flavored possibility semantics for classical logic in $[15,51] .^{22}$

\section{Discussion}

The preceding section summarized our case for making the explicit/implicit distinction and seeing where it leads. While this should suffice in practice, a number of critical concerns remain. Is the contrast just there, or does it have an explanation? Is it really different from received distinctions inside logic? Does the implicit/explicit

\footnotetext{
${ }^{21}$ For a recent extensive discussion of translatability and identity issues in logic, cf. the dissertation [38], and for new results on translations between modal logics, [39].

${ }^{22}$ Merges are a case where an over-zealous exclusive use of the implicit/explicit contrast makes no sense. Is intuitionistic modal logic explicit since it has modalities, or implicit because its underlying propositional base packages information that could be brought out by a further Gödel translation? One might also say that systems like this show how implicitness or explicitness in design can be a matter of degree.
} 
contrast have presuppositions that can be questioned, or that limit its applicability? We discuss such challenges, and see what remains of the contrast in the end.

Our first points address the nature of the distinction that we have been pursuing.

First-Person and Third-Person Views We have noted the existence of our contrast, but we have not offered an explanation of why it is there, or perhaps more to the point, of the co-existence of explicit and implicit approaches. It has been suggested by readers of this paper that the background may lie in well-known distinctions in logic and philosophy. One is that between reasoning with, from an internal first-person perspective, and reasoning about, from an external third-person perspective. Implicit logics might give the reasoning with, and explicit ones the reasoning about. But while this seems appealing, it does not quite fit with the way these systems function in practice. For instance, epistemic logic with operators can also be used in first-person mode by agents reasoning about their own information, and on the other hand, say, dynamic semantics has also been applied to third-person discourse. There may be a correlation between explicit design and a third-person stance, and implicit design with a first-person stance, but it does not seem very tight.

Object Language and Meta-Language Another distinction that seems congenial is that between object language and meta-language. We can often formalize the metalanguage of the semantics for a logical system in some other logic - the 'standard translation' for modal logic is a typical example, [18]. Is the meta-logic then the explicit version of an implicit object logic? In some cases this correlation fits well. One might view modal $S 4$ as formalizing a small, but significant part of the firstorder meta-theory of intuitionistic semantics. ${ }^{23}$ But even so, a complete identification of explicit logics with meta-theory inspired extensions of implicit logics does not fit universally. It is not at all clear, for instance, in which sense paradigmatic systems in philosophical logic such as doxastic or conditional logics are meta-logics of implicit systems - or for an example discussed at length in this paper, in which sense dynamic-epistemic logic is a meta-logic of dynamic semantics. Again, the object/meta distinction offers an interesting correlation, but no more than that.

Choosing Locally Co-existence means that both implicit and explicit stances have value, but particular areas may bring reasons for using one rather than the other. For instance, are there favored stances in human cognition? It has been claimed that natural language conveys much information implicitly, perhaps for ease of coding. Implicit logics would then model this reality directly, whereas explicit logics of information and agency are outside theorists' views of language. But this does not fit the empirical facts as I see them. Natural language is a medium where both stances occur, in the guise of what one might call participating versus observing modes in cognitive activity. A key empirical feature here is the universality of language. We switch

\footnotetext{
${ }^{23}$ Further telling illustrations of this meta-perspective occur at the interface of logic and games. The modaldynamic game logic of [45] formalizes part of the meta-theory of first-order evaluation games. Vice versa, game logics induce logic games, that is, implicit practices for analyzing their semantics - and this design cycle can even be iterated, cf. the research program developed in [14].
} 
between the two modes all the time, while firmly staying inside the same medium of communication. There may be local cognitive preferences between going explicit or implicit, but we doubt they can be justified in a sweeping manner.

Next we turn to critical points concerning the formulation of the implicit/explicit contrast, and its limitations. The first is a challenge to the way we described the balance in system design between setting deductive power and choosing vocabulary.

The Ubiquity of New Language Design Our presentation may have suggested that new language design is a preserve of the explicit design stance. But this is not right. While many implicit logics focus on reinterpreting existing vocabulary and changes in valid consequence, in some cases, new vocabulary does get created, as in non-monotonic logics with new operators for minimizing along orderings, [11]. As observed in Section 4, there is a general mechanism at work here. In all cases of weakening existing logics by enriching model classes with new semantic primitives (ordering, admissibility, and the like), one can introduce new vocabulary for those primitives - even though this potential is often ignored, because of a conservative focus on giving a semantics for a fixed language given beforehand. However, this is just the sort of setting where our earlier analysis applies. If the new models have independent explanatory power, we can take them as a platform for design of logics, and pursue the same implicit/explicit contrast that we have proposed. ${ }^{24}$

Next, consider an evident methodological presupposition of all the comparative case studies presented in this paper: the setting was always model-theoretic.

The Challenge from Proof Theory Most of our discussion was focused on meaning and valid consequence, and we needed semantic common ground to compare the logical systems we were interested in. We also saw that this choice of semantic platform is not unique, introducing a perspective dependence to the implicit/explicit distinction. This variety is not necessarily harmful: the contrast can be made to work then in different settings. But there is a more important concern. Does our model-theoretic analysis transfer at all to a syntactic proof-theoretic paradigm?

There are severe challenges here. To mention just one, in a proof-theoretic perspective, given any logical system, it makes sense to look for natural inferential fragments that use less proof power for applications. In this way, we can start with an explicit logic and change its base calculus, without having a primary semantic motivation for doing so. In that setting, our contrast does not readily apply. ${ }^{25}$

But there is more. Proof-theoretically weaker logics often come with an extended vocabulary that has a purely combinatorial motivation of encoding proof patterns, cf.

\footnotetext{
${ }^{24}$ Of course, we are not suggesting that there is a unique, let alone algorithmic, method that covers all such settings. For instance, so-called generalized assignment semantics for weakened decidable first-order logic, [1], suggests various sorts of explicit-style new vocabulary for quantification, but this extension works differently from our modal operator examples so far. Moreover, its faithful translation into guarded fragments of standard first-order logic raises interesting further subtleties.

${ }^{25}$ Thomas Icard (p. c.) points out the case of 'natural logics', very small efficient fragments of logical systems, as a form of design where it is hard to contrast implicit and explicit features. For such small fragments, there is often no difference between classically valid and non-classical consequence.
} 
the product or residual operations in the substructural logics of [48]. To fit this into a model-theoretic perspective, one might introduce objects close to syntax as the relevant universe, as is done in completeness proofs for some substructural logics, and consider design choices for how to talk and reason about these objects. More generally, historically, even purely proof-theoretically motivated systems often acquire a model-theoretic semantics afterwards, and then our analysis applies again. Once a plausible semantics has been found for a proof-theoretically motivated alternative logic, one can usually find an explicit counterpart after all. An illustration was given in Footnote 16 in connection with resource logics.

Even so, finding a purely proof-theoretic companion, if one exists at all, for the general explicit/implicit contrast of this paper remains an open problem.

Aside: The Challenge from Algebraic Logic Similar points can be made about the algebraic perspective on logical systems. Again, it is not clear if our contrast applies. Algebraic logics arise by fixing some signature, choosing relevant equational validities, and exploring the universe of algebras satisfying these. No principled design alternative suggests itself in this setting. However, our contrast will reemerge once we find representation theorems that connect algebras with model-theoretic structures. Once we have these, our implicit/explicit contrast will come up again in the design of logics for the latter, that can then be algebraized again, and so on. ${ }^{26}$

Biased Terminology? The terms 'explicit' and 'implicit' have their uses, but in our examples, they reflected semantic settings where logical systems are compared with respect to some shared topic - which need not always exist. Alternatives such as 'extensionist' versus 'revisionist' might seem more neutral, but these would reflect the standpoint of classical logic as a point of departure, which seems biased.

Perhaps, in the end, the neutral perspective, and also the best way of taking the issues raised in this paper is one of degree in setting expressive and deductive power of logical systems. Where do we put the balance of vocabulary and inference engine when designing such systems, and what are the fruitful available options?

\section{Conclusion}

We have identified what we see as a significant methodological contrast running through logic, between implicit and explicit stances. We use the word 'stance' here, and not 'system', because we do not identify logic with a family of formal systems. Some logical systems can indeed be called implicit or explicit, but the contrast as we have discussed it also applies to broader working habits in analysis and design.

\footnotetext{
${ }^{26}$ Even so, there are intriguing challenges here that we cannot address in this paper. An algebraic approach to substructural logics can save some classical principles precisely by weakening the base logic. For instance, the associativity of truth maker semantics in Section 12 will cause undecidability in a classical propositional logic, but the relevant substructural associative algebras have a decidable theory, as in the residuated algebra approach of [26]. It would be of interest to compare such substructural algebras with explicit classical modal logics over the associated residuated frames.
} 
We have not hidden the fact that our contrast also has its limitations, and that it does not apply to all logical systems, or travel well from semantics to proof theory. The jury is still out on whether we can spring those boundaries.

Also, the contrast is not mathematically defined, and it leaves room for variants and it admits borderline cases. This would be a serious concern if we wanted precise classification and taxonomy, or algorithms turning implicit systems into explicit ones and vice versa. But to us, this slack just means that the contrast leaves room for creativity. Even in its current formulation, it reveals patterns running through the field of logic, and it suggests new questions of a conceptual and technical nature. We have shown this in concrete instances of system design and in translations between systems. So, awareness of our contrast means new interesting work to be done, and more generally, we see it as a force toward a better understanding of the coherence of logic, in systems and in working habits. But also, and perhaps more importantly, we have pointed out in several illustrations (dynamic semantics, logical pluralism, Quinean revision in logic, truth maker semantics) that awareness of the contrast has serious philosophical consequences, since it undercuts sweeping ideological views that are tacitly based on taking just one design option while ignoring others.

Acknowledgments This paper is based on lectures at the Hans Kamp 70 conference in Stuttgart 2010, Advances in Modal Logic, Copenhagen 2012, the Heyting Conference, Amsterdam 2013, the FEW Workshop on Formal Epistemology, Seattle 2017, as well as seminar presentations in Amsterdam, Beijing, New York, and Stanford. I thank members of these audiences for their feedback, as well as the referees of this paper, and Wesley Holliday, Thomas Icard, Steve Kuhn and Sonja Smets.

Open Access This article is distributed under the terms of the Creative Commons Attribution 4.0 International License (http://creativecommons.org/licenses/by/4.0/), which permits unrestricted use, distribution, and reproduction in any medium, provided you give appropriate credit to the original author(s) and the source, provide a link to the Creative Commons license, and indicate if changes were made.

\section{References}

1. Andréka, H., Bezhanishvili, N., Németi I., van Benthem, J. (2014). Changing a semantics: opportunism or courage? In Manzano, M., Sain I., Alonso, E. (Eds.) The life and work of Leon Henkin (pp. 307-337). Basel: Birkhaüser Verlag.

2. Artemov, S. (2007). Justification logic. Technical report TR-2007019. New York: CUNY Graduate Center.

3. Artemov, S., \& Protopopescu, T. (2016). Intuitionistic epistemic logic. Review of Symbolic Logic, 9 , 266-298.

4. Balbiani, P., \& Galmiche, D. (2016). About intuitionistic public announcement logic. In Beklemishev, L., Demri S., Maté, A. (Eds.) Proceedings advances in modal logic, Budapest 2016 (pp. 58-77). London: College Publications.

5. Baltag, A., \& Smets, S. (2008). A qualitative theory of dynamic interactive belief revision. In Bonanno, G., van der Hoek W., Wooldridge, M. (Eds.) Texts in logic and games (Vol. 3, pp. 9-58). Amsterdam University Press.

6. Baltag, A., \& Smets, S. (2011). Quantum logic as a dynamic logic. Synthese, 179:2, 285-306.

7. Baltag, A., \& Smets, S. (2017). Modeling correlated information change: from conditional beliefs to quantum conditionals. Soft Computing, 21(6), 1523-1535.

8. Bar-Hillel, Y., \& Carnap, R. (1953). Semantic information. The British Journal for the Philosophy of Science, 4:14, 147-157.

9. Barwise, J., \& Perry, J. (1983). Situations and attitudes. Cambridge: The MIT Press. 
10. Beall, J., \& Restall, G. (2006). Logical pluralism. Oxford: Oxford University Press.

11. van Benthem, J. (1989). Semantic parallels in natural language and computation. In Ebbinghaus, H.-D., et al. (Eds.) Logic colloquium. 1987 (pp. 331-375). NorthHolland, Amsterdam.

12. van Benthem, J. (1996). Modal logic as a theory of information. In Copeland, J. (Ed.) Logic and reality (pp. 135-168). Oxford: Clarendon Press.

13. van Benthem, J. (2011). Logical dynamics of information and interaction. Cambridge: Cambridge University Press.

14. van Benthem, J. (2014). Logic in games. Cambridge: The MIT Press.

15. van Benthem, J., Bezhanishvili, N., Holliday, W. (2017). A bimodal perspective on possibility semantics. Journal of Logic and Computation, 27:5, 1353-1389.

16. Benthem, J., \& Minica, S. (2012). Toward a dynamic logic of questions. Journal of Philosophical Logic, 41(4), 633-669.

17. Bezhanishvili, G., \& Holliday, W. (2017). A semantic hierarchy for intuitionistic logic to appear. In van Dalen, D., Klop, J.W., van Mill, J. (Eds.) Indagationes mathematicae, special issue on L. E. J. Brouwer.

18. Blackburn, P., Rijke, M., Venema, Y. (2000). Modal logic. Cambridge: Cambridge University Press.

19. Burgess, J. (1981). Quick completeness proofs for some logics of conditionals. Notre Dame Journal of Formal Logic, 22(1), 76-84.

20. Ciardelli, I., Groenendijk, J., Roelofsen, F. (2013). Inquisitive semantics: a new notion of meaning. Language and Linguistics Compass, 7(9), 459-476.

21. van Ditmarsch, H., Halpern, J., van der Hoek, W., Kooi, B. (Eds.) (2015). Handbook of logics for knowledge and belief. London: College Publications.

22. Dosen, K. (1985). Models for stronger normal intuitionistic modal logics. Studia Logica, 44, 39-70.

23. Eijck, J., \& de Vries, F.-J. (1992). Dynamic interpretation and hoare deduction. Journal of Logic, Language and Information, 1, 1-44.

24. Fernandez, D. (2006). A Polynomial translation of s4 into intuitionistic logic. Journal of Symbolic Logic, 71:3, 989-1001.

25. Fine, K. (2017). Truthmaker semantics. In Hale, B., Wright C., Miller, A. (Eds.) A companion to the philosophy of language, 2nd Edn. (pp. 556-577). Wiley On-Line Library.

26. Galatos, N., \& Jipsen, P. (2013). Residuated frames with applications to decida-bility. Transactions of the American Mathematical Society, 365, 1219-1249.

27. Gierasimczuk, N. (2010). Knowing one's limits, logical analysis of inductive inference dissertation. ILLC, University of Amsterdam.

28. Girard, P., Liu, F., Seligman, J. (2012). General dynamic dynamic logic. In Proceedings AIML Copenhagen.

29. Gödel, K. (1933). Eine Interpretation des intuitionistischen Aussagenkalküls. Ergebnisse eines Mathematischen Kolloquiums, 4, 39-40.

30. Goré, R., \& Thomson, J. (2017). A correct polynomial translation of s4 into intuitionistic logic' logic and computation group. Canberra: Australian National University.

31. Groenendijk, J., \& Stokhof, M. (1991). Dynamic predicate logic. Linguistics and Philosophy, 14(1), 39-100.

32. Halvorson, H. (2017). The invariant content of equivalent theories. Department of Philosophy, Princeton University, Talk at 6th CSLI Workshop on Logic, Rationality and Intelligent Interaction, Stanford.

33. Hamami, Y., \& Roelofsen, F. (2015). Logics of questions, preface to special issue. Synthese, 192(6), 1581-1584.

34. Hintikka, J. (1962). Knowledge and belief. Ithaca: Cornell University Press.

35. Holliday, W. (2012). Knowing what follows: epistemic closure and epistemic logic. Ph.D thesis, Department of Philosophy, Stanford University.

36. Hoshi, T. (2009). Epistemic dynamics and protocol information. Ph.D. thesis, Department of Philosophy, Stanford University (ILLC-DS-2009-08).

37. W. \& M. Kneale. (1962). The development of logic. Oxford: Oxford University Press.

38. Kocurek, A. (2018). What can you say? Measuring the expressive power of language. Dissertation, Group in Logic and the Methodology of Science, UC Berkeley. 
39. Kuhn, S., \& Weatherson, B. (2018). Notes on some ideas in Lloyd Humberstone's philosophical applications of modal logic. The Australasian Journal of Logic, 15, 1. https://doi.org/10.26686/aj1. v15i1.4072.

40. Levesque, H. (1984). A logic of implicit and explicit belief. In Proceedings AAAI-84, (pp. 198-202).

41. Lewis, D. (1973). Counterfactuals. Oxford: Blackwell.

42. McCarthy, J. (1980). Circumscription - a form of non-monotonic reasoning. Artificial Intelligence, 13, 27-39.

43. Miller, J., \& Moss, L. (2005). The undecidability of iterated modal relativization. Studia Logica, 97 , 373-407.

44. Olkhovikov, G. (2013). Model-theoretic characterization of intuitionistic propositional formulas. Review of Symbolic Logic, 6(2), 348-365.

45. Parikh, R. (1985). The logic of games. Annals of Discrete Mathematics, 24, 111-140.

46. Putnam, H. (1968). Is logic empirical? In Cohen, R., \& Wartofsky, M. (Eds.) Boston studies in the philosophy of science 5 (pp. 216-241). Dordrecht: Reidel.

47. Quine, W. (1951). Two Dogmas of empiricism. The Philosophical Review, 60, 20-43.

48. Restall, G. (2000). Substructural logics. London: Routledge.

49. Rodenhaüser, B. (2014). A matter of trust: dynamic attitudes in epistemic logic. Dissertation ILLC, University of Amsterdam.

50. Roelofsen, F., \& Cardelli, I. (2015). Inquisitive dynamic epistemic logic. Synthese, 192(6), 16431687.

51. Rumfitt, I. (2015). The boundary stones of thought. Oxford: Oxford University Press.

52. Shi, Ch. (2018). Reason to believe. Dissertation DS 2018-10 Institute for Logic, Language and Computation, University of Amsterdam.

53. Shoham, Y. (1988). Reasoning about change: time and change from the standpoint of artificial intelligence. Cambridge: MIT Press.

54. Stalnaker, R. (2006). On logics of knowledge and belief. Philosophical Studies, 128:1, 169-199.

55. Troelstra, A., \& van Dalen, D. (1988). Constructivism in Mathematics, Vol. 2. North-Holland, Amsterdam.

56. Veltman, F. (1996). Defaults in upyear semantics. Journal of Philosophical Logic, 25, 221-261. Also appeared in The Philosopher's Annual, 1997.

57. Yalcin, S. (2007). Epistemic modals. Mind, 116, 983-1026. 